\title{
TITGER - UMA PLANILHA ELETRÔNICA PARA SIMULAÇÃo DE TITULAÇÃO DE MISTURA DE COMPOSTOS POLIPRÓTICOS
}

\author{
André Fernando Oliveira*, Astréa F. de Souza Silva, Mário A. Tenan e Sérgio Lineu Olivo \\ Núcleo de Ciências Ambientais, Universidade de Mogi das Cruzes, Av. Cândido X. A. Souza, 200, \\ 08715-911 Mogi das Cruzes - SP, Brasil
}

Recebido em 31/3/05; aceito em 10/4/06; publicado na web em 26/9/06

\begin{abstract}
TITGER - AN ELECTRONIC WORKSHEET FOR SIMULATING THE TITRATION OF A MIXTURE OF POLYPROTIC SPECIES.
The aim of this paper is to present a simple way of treating the general equation for acid-base titrations based on the concept of degree of dissociation, and to propose a new spreadsheet approach for simulating the titration of mixtures of polyprotic compounds.

The general expression, without any approximation, is calculated a simple iteration method, making number manipulation easy and painless.

The user-friendly spreadsheet was developed by using MS-Excel and Visual-Basic-for-Excel. Several graphs are drawn for helping visualizing the titration behavior. A Monte Carlo function for error simulation was also implemented. Two examples for titration of alkalinity and McIlvaine buffer are presented.
\end{abstract}

Keywords: titration simulation; mixture of polyprotic species; acid-base titration.

\section{INTRODUÇÃO}

A titulação é uma técnica analítica clássica, e gradualmente seu uso foi sendo reduzido a um nicho de aplicações bem definido, devido ao desenvolvimento de outras técnicas analíticas mais rápidas e com menores limites de detecção. Entretanto, ela ainda representa uma ferramenta muito útil para auxiliar na compreensão de equilíbrios químicos, principalmente o equilíbrio ácido-base, pois é possível observar o comportamento das substâncias em diferentes concentrações, na presença de outras.

O problema do ensino de cálculo de titulação ácido-base está relacionado, em parte, ao tratamento matemático exaustivo e pouco atraente para grande parte dos alunos. Este é também um grande problema associado ao ensino do equilíbrio em solução. Para que possa ser abordado sem o uso de computadores, o tratamento matemático usualmente contém várias aproximações. Algumas aproximações são muito severas, obrigando sua verificação caso a caso, o que demanda um tempo que poderia ser utilizado para a solução de problemas mais próximos da realidade.

Deve-se deixar claro, entretanto, que não se faz aqui apologia à extinção do tratamento que envolve as aproximações citadas, mas que o uso de programas versáteis, que permitam que os cálculos sejam realizados sem aproximações, ajudará a abordar algumas questões de interesse em maior profundidade, ou mesmo auxiliar na comprovação dos resultados obtidos por aproximações.

No caso do cálculo de titulações, as propostas de equações gerais da curva de titulação preocupam-se com a solução matemática (analítica) das equações, o que determina maior complexidade, além de serem de difícil generalização $0^{1-10}$. Vale ressaltar, entretanto, a existência de programas para cálculo de titulações, mas que ou apresentam uma interface menos atraente ou têm menos possibilidade para tratamento dos resultados ${ }^{11-13}$.

Este trabalho tem dois objetivos: apresentar uma forma mais simples de tratar a equação geral de titulações ácido-base e, apresentar o TitGer, uma planilha elaborada no Microsoft Excel 97, que

*e-mail: ferqa@umc.br permite a simulação de curvas de titulação envolvendo uma solução formada por até três substâncias polipróticas (com até quatro sítios doadores ou aceptores de prótons), e de uma solução titulante, formada por até duas substâncias, também com possibilidade de até quatro sítios protonáveis. Esse último recurso permite avaliar, por ex., a influência de um contaminante na solução titulante. Além disso, é possível introduzir um erro aleatório, com distribuição normal em qualquer variável e avaliar seu efeito sobre outros parâmetros da titulação. A curva de distribuição das espécies envolvidas é calculada também e seu comportamento durante a titulação pode ser observado graficamente. Cópias da planilha TitGer tratadas neste artigo podem ser obtidas gratuitamente na internet ${ }^{14}$.

A escolha do software Microsoft Excel deve-se a vários fatores. O primeiro, é que há uma ampla presença desse programa nos computadores pessoais, de universidades e de empresas. Essa planilha permite a construção de macros, ou seja, é possível programar em Visual Basic para Aplicações. Além disso, esse software permite a construção de gráficos e a realização de outros cálculos utilizandose diretamente o resultado da simulação e apresenta uma interface muito amigável. Além dessas vantagens, os dados obtidos podem ser facilmente transferidos a outros programas computacionais, tais como o Microcal Origin ${ }^{15}$, dentro do qual todo o TitGer pode ser aberto e executado.

Com o TitGer, atualmente na versão 1.8, é possível, além da simulação da curva de titulação, observar suas derivadas primeira e segunda, e verificar o comportamento da titulação através dos gráficos de Sorensen, Gran I e Gran II. Calcula-se também a distribuição das espécies envolvidas durante a titulação (via cálculo dos graus de dissociação), de modo que os diagramas de grau de dissociação versus volume ou $\mathrm{pH}$ do meio podem ser avaliados.

\section{Cálculo}

A dedução da equação geral da titulação para o estudo de equilíbrio em solução é interessante por utilizar quatro conceitos importantes: balanço de massa, grau de dissociação, balanço de carga e constantes de equilíbrio. 
O balanço de carga é uma expressão do princípio da eletroneutralidade, ou seja, a soma das concentrações das cargas positivas deve ser igual à soma das concentrações de cargas negativas.

$[+]=[-]$

Lembrando que a contribuição de carga de um íon $i$ é igual ao produto de sua concentração ([1́on $]$ ]) pela sua carga $\left(\mathrm{q}_{\mathrm{i}}\right)$, o balanço de carga também pode ser escrito como:

$$
\sum_{i} q_{i}\left[\text { ion }_{i}\right]=0
$$

Por ex., o balanço de carga de uma solução aquosa de carbonato de sódio será:

$\left[\mathrm{Na}^{+}\right]+\left[\mathrm{H}^{+}\right]=\left[\mathrm{OH}^{-}\right]+\left[\mathrm{HCO}_{3}^{-}\right]+2 \cdot\left[\mathrm{CO}_{3}^{2-}\right]$

ou, equivalentemente,

$\left[\mathrm{Na}^{+}\right]+\left[\mathrm{H}^{+}\right]-\left[\mathrm{OH}^{-}\right]-\left[\mathrm{HCO}_{3}^{-}\right]-2 \cdot\left[\mathrm{CO}_{3}^{2-}\right]=0$

A relação entre a concentração de um íon hipotético A e sua concentração total (ou analítica) $C_{T}$ é definida pelo grau de dissociação,

$\alpha_{A^{-}}=\frac{\left[A^{-}\right]}{C_{T}}$

A grande utilidade desse parâmetro é que ele pode ser calculado para qualquer espécie química, a partir das constantes de equilíbrio ácido-base e do $\mathrm{pH}$ do meio. Ou seja, não é necessário ter-se conhecimento da concentração, em solução, do íon ou de sua concentração total (analítica). Devido à dependência do grau de dissociação com o pH é possível construir curvas de distribuição de espécies, independente de outras espécies presentes no meio. Por ex., as curvas de distribuição de espécies para o sistema do ácido acético/acetato, descrito pela equação de equilíbrio

$$
\mathrm{CH}_{3} \mathrm{COOH}_{(\mathrm{aq}} \rightleftharpoons \mathrm{CH}_{3} \mathrm{COO}_{(\text {(aq) }}^{-}+\mathrm{H}_{(\text {(aq) }}^{+}
$$

e para o sistema do carbonato, descritos pelos equilíbrios

$\mathrm{H}_{2} \mathrm{CO}_{3(\mathrm{aq})} \rightleftharpoons \mathrm{HCO}_{3}^{-}{ }_{(\mathrm{aq})}+\mathrm{H}^{+}{ }_{(\mathrm{aq})}$

$\mathrm{HCO}_{3 \text { (aq) }}^{-{ }_{(\mathrm{aq})}} \rightleftharpoons \mathrm{CO}_{3}{ }^{2-}{ }_{\text {(aq) }}+\mathrm{H}^{+}{ }_{\text {(aq) }}$

são apresentadas na Figura 1.

As Equações para cálculo do grau de dissociação das espécies são obtidas considerando-se o balanço de massa e as equações das constantes de dissociação de ácido envolvidas. A dedução dessas equações pode ser encontrada em livros-texto ${ }^{7,8}$. Ainda que pareçam excessivamente matemáticas, as expressões podem ser facilmente implementadas em planilhas do próprio Excel ou com outros programas computacionais, tais como AlfaCalc, etc. ${ }^{14}$. Assim, o grau de dissociação $\left(\alpha_{\mathrm{i}}\right)$ de uma espécie química $i$ pode ser calculado a partir da Equação 6:

$\alpha_{i}=\frac{\left[H_{n-i} A^{q-i}\right]}{C_{T}}=\alpha_{o} \frac{K_{1} K_{2} \ldots K_{i}}{\left[H^{+}\right]^{i}}$

onde

$\frac{1}{\alpha_{o}}=1+\frac{K_{1}}{\left[H^{+}\right]}+\frac{K_{1} K_{2}}{\left[H^{+}\right]^{2}}+\ldots+\frac{K_{1} K_{2} \ldots K_{n}}{\left[H^{+}\right]^{n}}$
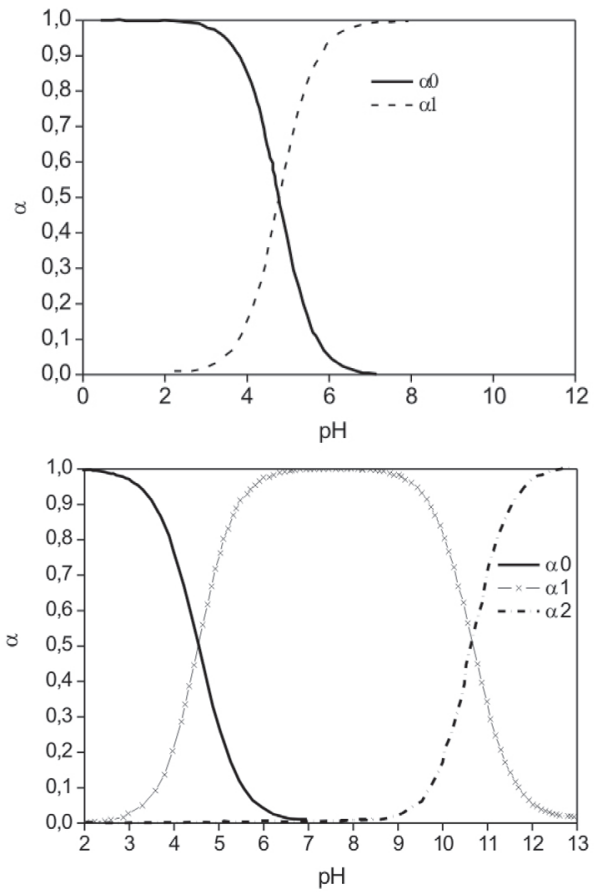

Figura 1. Diagramas de distribuição de espécies para: (acima) o sistema ácido acético/acetato; (abaixo) sistema ácido carbônico /bicarbonato / carbonato. $O \alpha_{o}$ é o grau de dissociação da espécie mais protonada

n é o número de prótons ionizáveis da espécie totalmente protonada, q é a carga da espécie totalmente protonada, $\mathrm{K}_{1}, \ldots . \mathrm{K}_{\mathrm{n}}$ são as constantes de equilíbrio para as espécies em questão.

Os graus de dissociação apresentam inúmeras aplicações, auxiliando na explicação da influência do pH no fator de capacidade em cromatografia $^{16,17}$, na mobilidade eletroforética aparente e na carga líquida $^{18-20}$, na solubilidade de sais e gases ${ }^{6,7}$, na capacidade de complexação ${ }^{7,8}$, na cinética de reações ${ }^{21}$, etc.

De posse das expressões do balanço de carga, Equação 2, e dos graus de dissociação das espécies envolvidas, Equação 6, pode-se escrever a equação da titulação ácido-base.

A titulação considerada será formada por um volume inicial $\left(\mathrm{V}_{\mathrm{o}}\right)$ de uma mistura de vários compostos $\mathrm{j}$ (titulados), sobre o qual serão adicionadas alíquotas (V) de uma solução titulante (também formada pela mistura de até dois compostos).

Em cada ponto, ou seja, após a adição de cada alíquota de titulante $\left(\mathrm{C}_{\mathrm{Nj}}\right)$ sobre o volume inicial de titulado, a concentração dos contra-íons será alterada devido à diluição no meio. Para os titulantes $\left(\mathrm{C}_{\mathrm{Dj}}\right)$, a concentração no meio após cada adição da solução contendo uma concentração estoque $\left(\mathrm{C}_{\mathrm{Dj}}{ }^{\text {estoque }}\right)$ é:

$C_{D j}=\frac{C_{D j}^{\text {estoque }} V}{V_{o}+V}$

enquanto que, para os titulados, a concentração é obtida pela expressão:

$C_{N j}=\frac{C_{N j}^{\text {estoque }} V_{o}}{V_{o}+V}$

Como a titulação envolve o balanço de carga da solução contendo diversos compostos, uma forma mais simples de descrevê-la é considerar a contribuição $\left(\mathrm{Q}_{\mathrm{j}}\right)$ de cada composto $j$, ou seja, a soma das concentrações das cargas do contra-íon $\left(\mathrm{C}_{\mathrm{ci}} \cdot \mathrm{q}_{\mathrm{ci}}\right)$ e das diversas 
espécies envolvidas:

$$
\left.Q_{j}=C_{c i} q_{c i}-\sum q_{i} \cdot\left[H_{n-i} A^{q-i}\right]\right)
$$

que pode ser reescrita, considerando-se o grau de dissociação de cada espécie

$$
Q_{j}=C_{c i} q_{c i}\left(1-\sum q_{i} \cdot \alpha_{i}\right)
$$

Assim, em qualquer titulação ácido-base, a expressão geral será:

$$
\left[\mathrm{H}^{+}\right]-\left[\mathrm{OH}^{-}\right]+\sum_{j} Q_{j}=0
$$

Como se vê na Equação 10, a expressão geral da titulação é função de várias constantes e da concentração de $\mathrm{H}^{+}$apenas, para cada volume de titulante adicionado ao meio.

É claro que a resolução analítica dessa equação torna-se bastante difícil quando se considera uma solução com mais de um composto. Portanto, o emprego de métodos numéricos torna-se muito interessante.

Dentre os vários métodos para obtenção de uma solução numérica $^{22}$ foi escolhido o método iterativo por aproximações sucessivas, que é bastante simples e facilmente implementável ${ }^{8}$. Embora o número de iterações necessárias possa ser muito grande comparado ao de outros algoritmos ${ }^{22}$, esta desvantagem não é significativa, considerando-se a taxa de processamento dos computadores atuais.

De acordo com esse método, um valor inicial de $\mathrm{pH}$ é testado na Equação 10 para um dado volume de titulado. Se este valor não for o correto, o resultado para o primeiro membro da Equação 10 será diferente de zero. O programa realiza, então, uma segunda tentativa, com outro valor de $\mathrm{pH}$ (valor inicial mais um incremento pré-definido). $\mathrm{O}$ processo é repetido até que o resultado tenha o sinal invertido em relação aos valores anteriores, indicando que se passou pela solução da Equação 10. A partir desse passo, o incremento é alterado para metade do valor anterior e com o sinal também trocado. O processo é repetido até que o incremento seja inferior a um valor limite, que representa a incerteza no resultado. No caso do TitGer 1.8, o valor inicial testado foi bem baixo (-1) e o valor limite do incremento foi de 0,0001. Esses valores podem ser alterados pelo usuário.

\section{TitGer 1.8}

A planilha TitGer 1.8 foi desenvolvida para que os conheci- mentos sobre as técnicas de programação ou sintaxe dos programas matemáticos não sejam essenciais para a compreensão dos equilíbrios ácido-base.

O programa foi desenvolvido com auxílio da linguagem Visual Basic para Excel. Seu algoritmo simples pode ser acompanhado por qualquer leitor interessado no assunto.

O TitGer é composto por várias pastas ("worksheets"), ou seja, várias telas do Excel, a citar 'Principal', 'Gráficos', 'Linearização', 'DistrEspécies', 'Dados' e 'Equações', visando facilitar seu uso e tornar cada tela menos poluída visualmente. Apenas algumas pastas serão discutidas neste artigo.

Na pasta 'Principal' (Figura 2) o usuário deve introduzir, nos campos pertinentes (células da planilha) as informações necessárias à simulação, tais como as concentrações analíticas dos compostos, tanto na solução titulante (células B10 e/ou F10) quanto na solução titulada (B22 e/ou F22 e/ou J22), volume inicial da solução titulada (C3) e incremento de volume da solução titulante (C5). Na forma "default" da planilha, os valores de volume de titulante a serem adicionados são calculados por funções já inseridas diretamente nas células adequadas (a partir da E37), a partir do incremento de volume definido na célula B5. Na Figura 2, o valor do incremento foi 0,25 $\mathrm{mL}$. Este formato pode, entretanto, ser alterado pelo usuário.

Para facilitar a escolha dos compostos envolvidos na titulação e as informações intrínsecas relacionadas aos mesmos, a planilha foi implementada com caixas de seleção ('combo-box'). As 'combobox' apresentam, em forma de listas, as espécies disponíveis (por ex., ácido carbônico, bicarbonato ou carbonato de sódio) e com sua seleção, os valores das constantes de equilíbrio e número de sítios de protonação e da carga da espécie são conveniente e automaticamente colocados na planilha (Figura 2).

A versão apresentada permite que a solução do titulante seja formada por dois compostos e o titulado, por três. Todos eles podem ter até quatro constantes de equilíbrio.

Para permitir a descrição adequada de cada espécie na simulação, consideram-se dois tipos possíveis de sítios protonáveis em uma espécie química: aqueles que, protonados, não têm carga (na planilha foi utilizada a expressão genérica '-COOH'), tais como carboxilatos, nitrito, sulfito, etc.; e aqueles que, protonados, adquirem carga positiva (chamados na planilha '- $\mathrm{NH}_{2}$ '), tais como as aminas, amônia, etc.

Tanto os valores de pKa, quanto os números dos sítios de cada composto apresentados nas caixas de seleção estão em um outra pasta, chamada 'Dados', que representa o banco de dados utilizado, cujos parâmetros podem ser alterados pelo usuário ou até mesmo

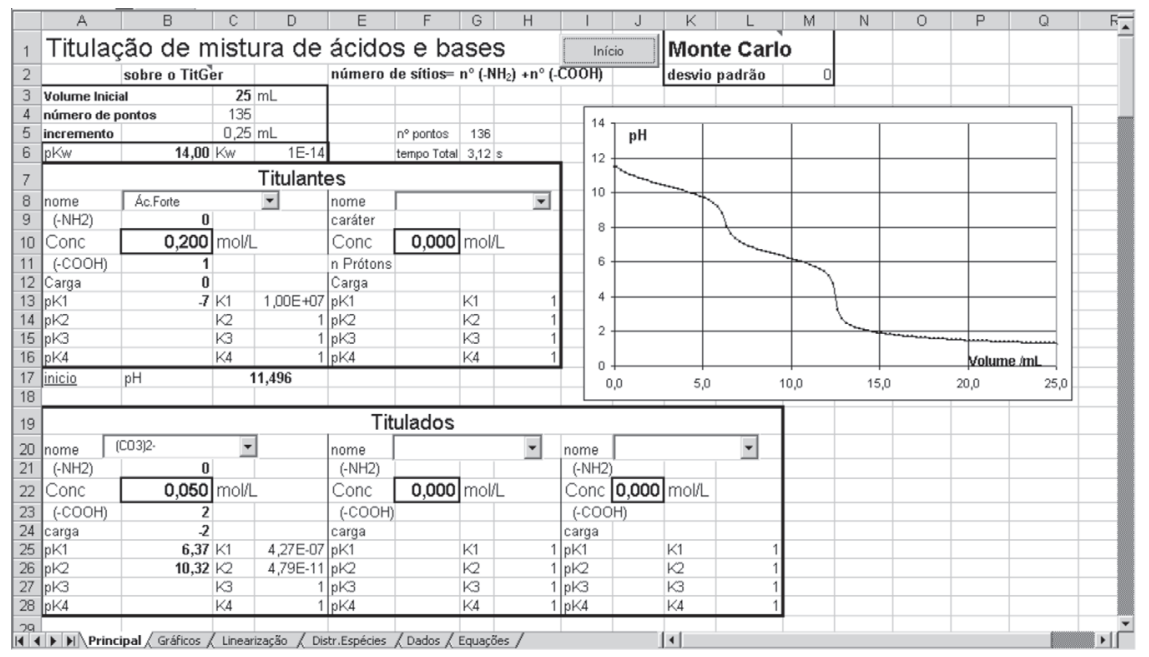

Figura 2. Tela da pasta principal do programa TitGer. Condições para simulação da titulação de $25 \mathrm{~mL}$ de carbonato 0,050 mol/L com alíquotas de 0,25 mL de ácido forte $0,200 \mathrm{~mol} / \mathrm{L}$ 
novas espécies e/ou parâmetros podem ser acrescentados.

A macro (programa em Visual Basic for Excel) lê os volumes listados na coluna 'A' (a partir da célula A37, na pasta 'Principal') e fornece os valores de $\mathrm{pH}$ calculados na coluna ' $\mathrm{C}$ '.

A apresentação dos dados (volume e pH) em colunas do Excel permite a fácil manipulação dos dados, como apresentação de gráficos da titulação (presente na Pasta 'Principal' e na Pasta 'Graficos'), da derivada primeira e segunda e do poder tamponante (já definidos na pasta 'Gráficos' - Figura 3). Alguns métodos de linearização, método de Sorensen ${ }^{23,24}$ e de Gran I ${ }^{25}$ e duas variações do método de Gran II $^{26,27}$ foram colocados em outra pasta (Pasta 'Linearização') e são apresentados na Figura 4. As regiões lineares nos gráficos podem ser ajustadas por modelos de regressão linear disponíveis em várias funções do Excel ('proj.lin', linhas de tendência, etc.).
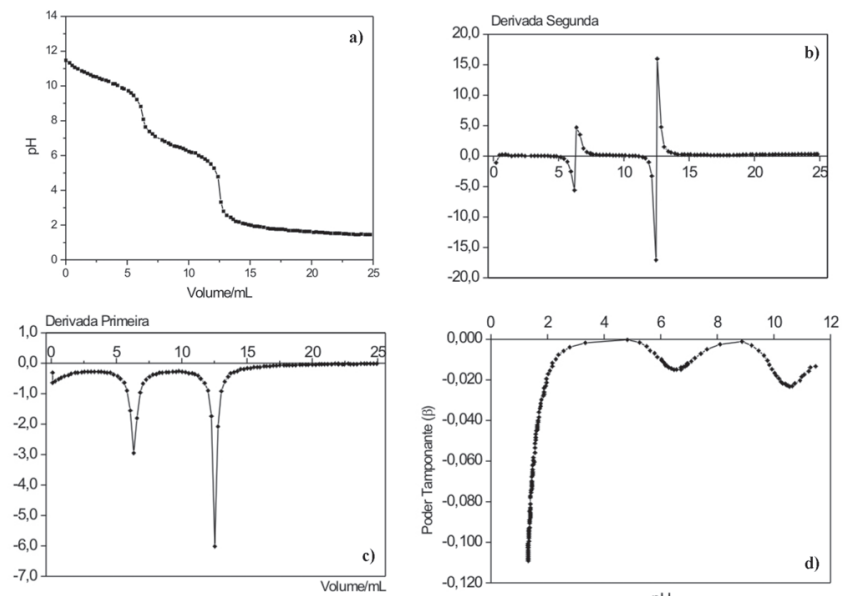

Figura 3. Gráficos apresentados na pasta gráficos (condições definidas na figura 2); a) curva de titulação; b) gráfico de derivada segunda; c) gráfico de derivada primeira; d) gráfico do poder tamponante
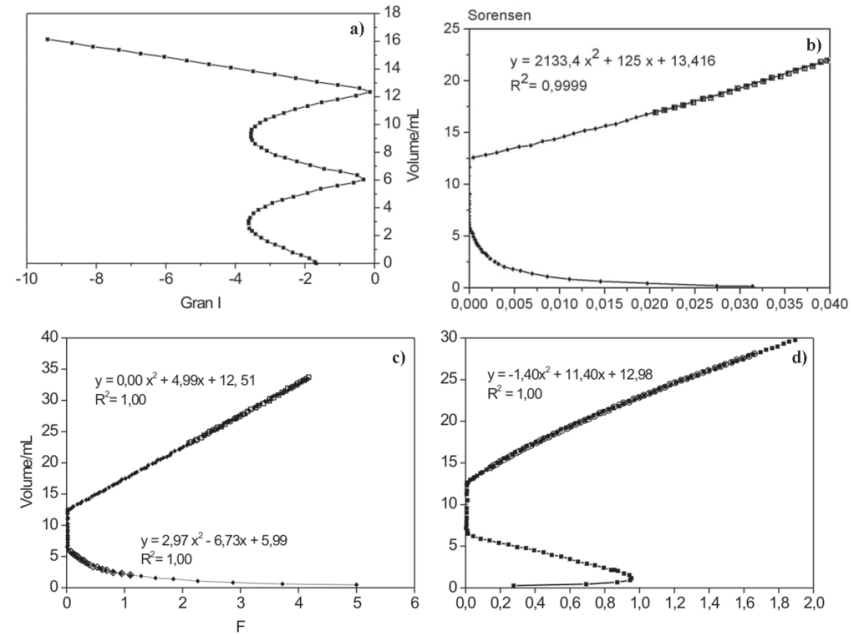

Figura 4. Métodos de linearização de uma curva de titulação de carbonato com ácido forte; a) Método de Gran I; b) método de Sorensen; c e d) duas variações do Método de Gran II

\section{Distribuição de espécies}

A curva de distribuição de espécies é um gráfico muito útil para visualização do comportamento das diversas espécies de um composto em função do $\mathrm{pH}$ (Figura 1b). O programa apresenta esse gráfico no intervalo de $\mathrm{pH}$ em que ocorre a titulação para cada composto titulado.
A compreensão da curva de titulação é muito facilitada pela sobreposição da curva de distribuição de espécies ${ }^{28}$. A Figura 5 foi gerada automaticamente no TitGer na pasta 'Distr.Espécies' e é um exemplo dessa sobreposição. Vê-se claramente que a quantidade de bicarbonato aumenta com o consumo de carbonato até o ponto de equivalência $(12,5 \mathrm{~mL})$ e diminui após esse ponto com a adição de mais titulante, até o segundo ponto de equivalência (25 $\mathrm{mL}$ ). Os pontos de cruzamento das curvas de distribuição das espécies mostram que as regiões de maior tamponamento do meio ocorrem nos valores de $\mathrm{pH}$ numericamente próximos dos valores dos $\mathrm{pK}_{\mathrm{a}}$ do sistema em estudo $(6,25$ e $18,75 \mathrm{~mL})$.

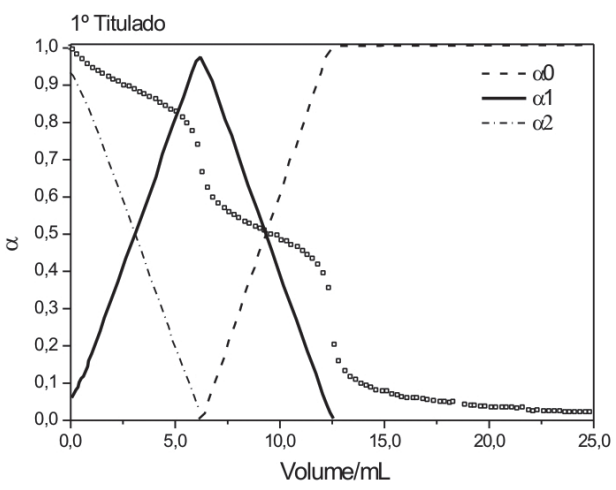

Figura 5. Sobreposição dos graus de distribuição de espécie sobre a curva de titulação normalizada (condições da titulação definidas na Figura 2). $\alpha_{\alpha}$, representa o grau de distribuição do ácido carbônico; $\alpha_{1}$, bicarbonato e $\alpha_{2}$, carbonato

\section{Aplicações}

Para demonstrar a potencialidade do programa são apresentados a seguir dois exemplos de aplicação: titulação de alcalinidade em águas e preparo do tampão de McIlvaine, sendo que este último não pode ser considerada uma titulação no sentido literal.

Para realizar a simulação da titulação de alcalinidade em águas, ou seja, para uma mistura de carbonato e de bicarbonato de sódio, é necessário considerar cada espécie como um composto, com sua respectiva concentração 'analítica'. Desta maneira, na pasta Principal, no campo referente à solução titulante, é selecionado o ácido forte e inserida sua concentração $(0,200 \mathrm{~mol} / \mathrm{L})$ e no campo titulado, as espécies carbonato e bicarbonato foram selecionadas em cada combo-box, ambas com concentrações iguais a $0,05 \mathrm{~mol} / \mathrm{L}$.

Na Figura 6 são apresentadas as curvas obtidas para titulação do carbonato sozinho e aquela com o carbonato na presença de bicarbonato.

O programa TitGer pode ser aplicado também para estudo de soluções tampão, tanto na determinação do $\mathrm{pH}$ quanto na avalia-

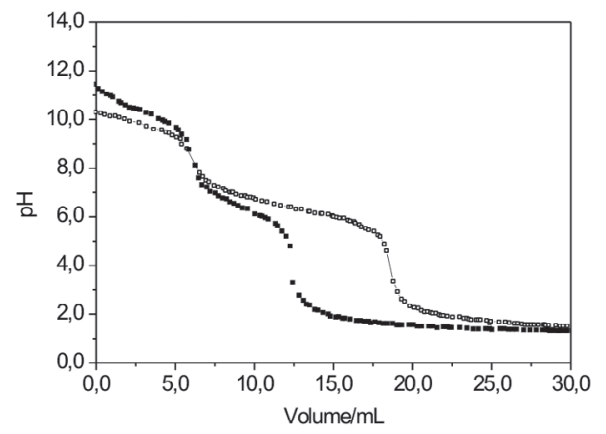

Figura 6. Comparação entre a titulação de carbonato $0,05 \mathrm{~mol} / \mathrm{L}(\mathbf{\square})$ e da mistura carbonato e bicarbonato $(0,05 \mathrm{~mol} / \mathrm{L}$ cada $)(\square)$, com ácido forte $0,20 \mathrm{~mol} / \mathrm{L}$ 
ção da influência do $\mathrm{pH}$ inicial e/ou da concentração do tampão sobre seu poder tamponante na presença de ácidos ou bases fortes.

Na Figura 7 é apresentada uma forma para calcular o tampão de McIlvaine, pela mistura de fosfato dibásico de sódio $0,5 \mathrm{~mol} / \mathrm{L}$ sobre $50 \mathrm{~mL}$ de ácido cítrico $0,5 \mathrm{~mol} / \mathrm{L}$. Devido aos valores próximos de pKa do ácido cítrico, é difícil calcular as proporções desse tampão por meio de aproximações. Neste ponto é interessante ressaltar que a versão atual do TitGer não faz correções para a força iônica do meio e para os coeficientes de atividade. A forma mais simples é considerar a força iônica do meio constante e selecionar os valores de pKa para esta condição.

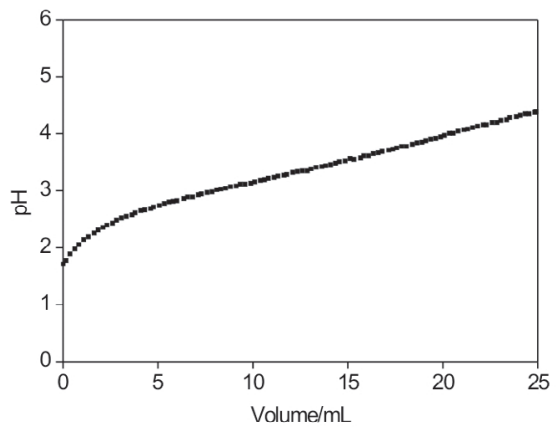

Figura 7. Adição de $\mathrm{Na}_{2} \mathrm{HPO}_{4}$ 0,5 mol/L sobre $20 \mathrm{~mL}$ de ácido cítrico 0,5 mol/L para preparo do tampão de McIlvaine

\section{Outras potencialidades}

O programa pode também simular a titulação quando se emprega um titulante contaminado como, por ex., uma solução de hidróxido de sódio $0,1 \mathrm{~mol} / \mathrm{L}$ contaminada com carbonato. No TitGer isso pode ser realizado, uma vez que há espaço para dois compostos na solução titulante. Desta forma, as diferenças, tanto no perfil da curva de titulação quanto no valor de equivalência em sistemas sem o carbonato ou em diferentes concentrações do mesmo, podem ser obtidas, simulando-se uma situação de cada vez.

Além disso, com o desenvolvimento da metrologia química, a necessidade de avaliação de erros nos procedimentos também tem aumentado. Uma forma elegante de avaliar o erro de uma variável na titulação é introduzir um erro aleatório (usualmente com distribuição normal) nessa variável e observar seu efeito na resposta (ponto de equivalência, de viragem, etc.). $\mathrm{O}$ efeito de indicadores pode ser avaliado pela introdução de dados do indicador, tais como concentração, pKa e sítios de protonação.

Para a introdução desse erro aleatório, o TitGer tem uma função chamada MC, que gera um número aleatório com distribuição normal de média zero e desvio-padrão igual a um valor definido pelo usuário (quando igual a zero, não há erro aleatório), baseada no método de Monte Carlo no algoritmo de Marsaglia, Box e Muller ${ }^{29}$. Essa função pode ser somada a qualquer variável da titulação, ou seja, pode ser adicionada a qualquer célula (valores de $\mathrm{pK}_{\mathrm{a}}$, de volumes, etc.). $\mathrm{Na}$ forma "default" do programa, essa função é adicionada ao volume que será lido pelo programa. Os gráficos, por sua vez, são elaborados vinculando-os à coluna cujos volumes de titulante não estão somados aos números aleatórios (coluna 'E', a partir da célula E37). Assim, a influência do erro no volume adicionado pode ser observada.

Outra aplicação é a avaliação de métodos de determinação de constantes de dissociação e pontos de equivalência propostos na literatura $^{30-34}$.

\section{CONCLUSÕES}

Neste trabalho é apresentada uma forma simples da equação geral de titulação ácido-base de mistura de polipróticos, utilizando o conceito de grau de dissociação.

A planilha TitGer 1.8 permite a simulação de titulações e avaliar o comportamento de mistura de compostos e contém formas de tratamento das curvas obtidas, tais como métodos de linearização e curvas de distribuição de espécies em função do $\mathrm{pH}$ e do volume de titulante. A planilha permite também a introdução na simulação de erros aleatórios com distribuição normal. São apresentados exemplos de titulações de carbonato e bicarbonato e o preparo do tampão de McIlvaine (citrato e fosfato).

O TitGer 1.8 é uma ferramenta facilitadora de estudos de titulações e equilíbrios ácido-base e pode auxiliar no ensino de equilíbrio químico nas diferentes áreas da química, tais como química analítica e química ambiental.

\section{AGRADECIMENTOS}

Ao auxílio financeiro da FAPESP e FAEP/UMC.

\section{REFERÊNCIAS}

1. Moura, D. R.; Campos-Filho, F. F.; Quim. Nova 1988, 11, 182.

2. Ivaska, A.; Nagypa, I.; Talanta 1980, 27, 721.

3. Scott, P.R.; Educ. in Chem. 1985, March, 40.

4. Moisio, T.; Heikonen, M.; Fresenius J. Anal. Chem. 1996, 356, 461.

5. Bos, M.; Anal. Chim. Acta 1979, 112, 65.

6. Stumm, W.; Morgan, J. J.; Aquatic Chemistry, $3^{\text {rd }}$ ed., Wiley-Interscience: New York, 1996.

7. Harris, D. C.; Quantitative Chemical Analysis, $5^{\text {th }}$ ed., Freeman and Co: New York, 1998.

8. Guenther, W. B.; Química Quantitativa-medições e equilíbrio, Edgar Blucher Ltda: Rio de Janeiro, 1972.

9. Levie, R.; J. Chem. Educ. 1970, 20.

10. Paniago, E. B.; Quim. Nova 1991, 14, 9.

11. Jurs, P. C.; Isenhour, T. L.; Wilkins, C. L.; BASIC Programming for chemists- an Introduction, Wiley-Intersciences: New York, 1987.

12. http:/alquemy.iq.usp.br//pub/tunelando/curtipot.zip, acessada em Julho 2005.

13. http:/allquemy.iq.usp.br/pub/tunelando/titratio.zip, acessada em Julho 2005.

14. http://www.umc.br/ ferqa/utils; http://ferqa.sites.uol.com.br/utils, acessadas em outubro 2005.

15. Microcal $^{T M}$ Origin versão 6.0; Microcal Software, Inc. Northampton, 1999.

16. Valkó, K.; Separation Methods in Drug Synthesis and Purification Elsevier: Amsterdam, 2000, p. 195.

17. Andrei, C. C.; Ferreira, D. T.; Faccione, M.; Faria, T. J.; Da Química Medicinal à química combinatória e modelagem molecular - um curso prático, Manole: Barueri, 2003, p. 39.

18. Alfenas, A.C., ed.; Eletroforese de isoenzimas e proteínas afins, Ed. UFV: Viçosa, 1998, p. 26-36.

19. Kuhn, R.; Hoffstetter-Kuhn, S.; Capillary electrophoresis- principles and practice, Springer-Verlag, Berlin, 1993.

20. Baker, D. L.; Capillary electrophoresis, Wiley and Sons: New York, 1995 , p.164.

21. Stumm, W.; Morgan, J. J.; Aquatic Chemistry, $3^{\text {rd }}$ ed., Wiley-Interscience: New York, 1996, p. 696.

22. Press, W. H.; Flannery, B. P.; Teukolsky, S. A.; Vetterling, W. T.; Numerical Recipes - the art of scientific computing, Cambrigde University Press: Cambrige, 1986, cap. 9.

23. Thomas, J. F. J.; Lynch, J. J.; J AWWA 1960, 52, 259

24. Larson, T. E.; Henley, L.; Anal.Chem. 1955, 27, 851.

25. Gran, G.; Analyst 1952, 77, 661.

26. Gran, G.; Anal.Chim.Acta 1988, 206, 111.

27. Gran, G.; Acta Chem. Scand. 1950, 4, 559.

28. Skoog, D. A.; West, D. M.; Holler, F. J.; Analytical Chemistry - an Introduction, $5^{\text {a }}$ ed., Saunders: Fort Worth, 1990, p. 218.

29. Demas, J. N.; Demas, S. E.; Interfacing and Scientific Computing on Personal Computers, Allyn and Bacon: Massachusetts, 1990.

30. Akimoto, N.; Hanakuma, H.; Hozumi, K.; Anal. Sci. 1987, 3, 515.

31. Smit, H. C.; Meites, L.; Kateman, G.; Anal. Chim. Acta 1983, 153, 121.

32. Covington, A. K.; Robinson, R. A.; Sarbar, M.; Anal. Chim. Acta 1978, 100, 367.

33. Pehrsson, L.; Ingman, F.; Johansson, A.; Talanta 1976, 23, 769.

34. Pehrsson, L.; Ingman, F.; Johansson, A.; Talanta 1976, 23, 781. 